\title{
An Equipment Condition Warning Method Based on MEP in Smart Substation
}

\author{
Li Jiansheng, Zhou Zhicheng, Wu Yiming, Lu Yuncai, Wei Chao, Wu Peng \\ Jiangsu Electric Power Company Research Institute, Nanjing 211103, China
}

Keywords: condition assessment, K-means clustering, maximum entropy principle, Monte Carlo simulation

\begin{abstract}
To monitor the equipment condition changes, a warning method based on MEP (Maximum Entropy Principle) is proposed. First, the data is normalized and the data objects are divided into K clusters by K-means clustering algorithm. Second, the parameters are calculated and its probability density function is obtained by maximum entropy principle. Last, the new parameter data is compared with warning data to decide the condition changes. In this method, clustering operation can reduce the impacts of outside environments and probability density function based on maximum entropy principle avoids the short comings of subjective assumption. Monte Carlo simulations verify the effectiveness and accuracy.
\end{abstract}

\section{Introduction}

With the development of computer and sensor technology, a large number of secondary systems have successfully applied into power system, such as supervisory control and data acquisition system, distribution management system and the wide area measurement system. These devices acquire and record comprehensive data, but used insufficiently. Excavating the information to reflect the power network state, forecast future problems and take measures, has important significance to realize the safe operation of the grid [1-3].

The equipment operation performance is relative with the inter structure and the external environment. First, the parameters are clustered by K-means algorithm using environment temperature and load data. Second, the equipment state representation parameters are identified by electrical voltage and current information, and the parameter distribution is derived by maximum entropy principle. Last, the equipment condition changes are detected by comparing the new data with the warning value. Due to the clustering operation and the probability distribution calculated by maximum entropy principle, the detection results avoid subjective assumptions and are accurate.

\section{Equipment condition representation parameters}

With the increase of the operation time, the equipment material ages, leading to the operation performance decline, which can be reflected by equivalent circuit parameter changes. And the relationship of the measured electrical information and equipment structure can be expressed by formula (1). Here, $z, u$ and $i$ represents the equipment parameter, the measured voltage and current information. The influence of the external environment is represented by $E$.

$$
z=f(u, i, E)
$$

Take the transformer as an example, the equivalent circuit parameters are shown in figure 1.

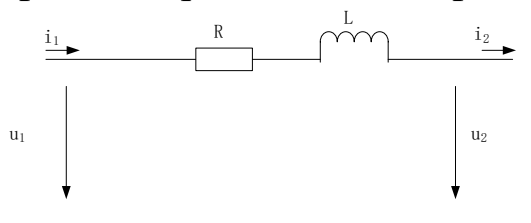

Fig.1 Equivalent circuit of transformers

The resistance $R$ representing transformer state can be calculated by (2). 


$$
R=\operatorname{real}\left(\frac{\dot{U}_{1}-\dot{U}_{2}}{\dot{I}_{2}}\right)
$$

Wherein, the superscript - represents the RMS data and real is the real part calculation operation.

In the operation process, the calculation value which can represent equipment condition is influenced by environment. To reduce the influence, a method based on clustering and MEP is presented.

\section{K-means clustering analysis and Probability density calculation}

\section{K-means clustering analysis}

Clustering analysis is one of the data mining technologies. And the commonly used methods contain K-means, K-medoids and others [12-13].

Assume there are $n p$ attributes and nobj objects, which are expressed by formula (3) and (4).

$$
\begin{aligned}
& \text { obj }=\left(p_{1}, p_{2}, \cdots, p_{n p}\right) \\
& {\left[\begin{array}{ccccc}
p_{11} & \cdots & p_{1 f} & \cdots & p_{1 n p} \\
\vdots & \vdots & \vdots & & \vdots \\
p_{n 11} & \cdots & p_{n 1 f} & \cdots & p_{n 1 n p} \\
\vdots & \vdots & \vdots & & \vdots \\
p_{\text {nobj1 }} & \cdots & p_{\text {nobjf }} & \cdots & p_{\text {nobjnp }}
\end{array}\right]}
\end{aligned}
$$

After the standardization of the primary data, the clustering analysis is carried out as follows.

Step 1: Select $K$ objects as the initial clustering centers.

Step 2: Calculate the Euclidean distance between the remaining objects and the clustering centers by formula (5).

$$
d\left(o b j_{n 1}, o b j_{n 2}\right)=\sqrt{\sum_{k=1}^{n p}\left|p_{n 1 k}-p_{n 2 k}\right|^{2}}
$$

Step 3: According to the distance value, put the object to the nearest cluster and recalculate each cluster center by formula (6).

$$
M_{k}=\frac{1}{n_{k}} \sum_{n 1=1}^{n_{k}} o b j_{n 1}
$$

Step 4: Repeat the above two steps until the criterion function converges. The criterion calculation is expressed as formula (7). $E_{K}{ }^{2}$ is the square error containing $K$ clusters and $e_{k}^{2}$ is the variance sum within the $k_{\text {th }}$ cluster.

$$
E_{K}^{2}=\sum_{k=1}^{K} e_{k}^{2}
$$

\section{Probability density calculation based on MEP}

The basic idea of MEP is to calculate the probability density ensuring the entropy maximum with only a partial knowledge [14-15].

Assume that the equivalent circuit parameter is expressed by $x$ and the probability value is $f(x)$. The maximum entropy model can be expressed by (8).

$$
\begin{array}{ll}
\max & H(x)=-\int_{R} f(x) \ln f(x) d x \\
\text { s.t. } & \int_{R} f(x) d x=1 \\
& \int_{R} f(x) x^{r} d x=a_{r}, r=1,2, \cdots, m
\end{array}
$$

Wherein, $H$ is the entropy and $R$ is the boundary value.

Construct the Lagrange equation as formula (9) and calculate it by (10). Here, $\lambda_{0} \sim \lambda_{\mathrm{m}}$ are the Lagrange multipliers. 


$$
\begin{gathered}
F(x)=H(x)+\left(\lambda_{0}+1\right)\left(\int_{R} f(x) d x-1\right)+ \\
\lambda_{1}\left(\int_{R} x f(x) d x-a_{1}\right)+\cdots+\lambda_{m}\left(\int_{R} x^{m} f(x) d x-a_{m}\right) \\
f(x)=\exp \left(\lambda_{0}+\sum_{r=1}^{m} \lambda_{r} x^{r}\right)
\end{gathered}
$$

\section{Equipment condition warning processes}

Based on the maximum entropy principle, the equipment condition warning processes are as follows.

(1) Analyze the external factors which are relative with the condition representation parameters, and deal with the data to form matrix.

(2) Calculate the distance and do clustering operation using $K$-means algorithm.

(3) Calculate the equipment parameters and the probability density function of the cluster containing the most samples.

(4) Set the warning value to detect the equipment state changes.

After the probability density function calculation, the warning value is derived according to the cumulative probability, which is expressed by formula (11).

$$
P=\int_{0}^{x_{w}} f(x) d x
$$

Select the proper data of $P$, and the corresponding data of $x_{w}$ is set to be warning data.

(5) Compare the new parameter sequences in the designated cluster with the alarm value. The criterion is shown in formula (12). Here, $x_{\text {new }}$ is the new parameter sequence and $n_{\text {new }}$ is its number. The threshold value is expressed by $\varepsilon$.

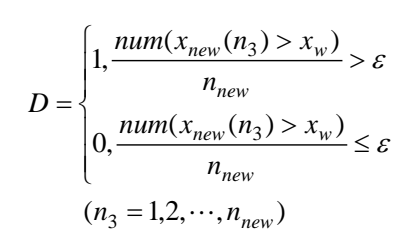

If $D$ is equal to 1 , the equipment condition is detected changed. Otherwise, repeat (2) - (5).

\section{Simulation verification}

Taking the transformer as an example, its condition changes detection by equivalent circuit parameters should reduce the environment and load fluctuation influence.

In this paper, the daily law of load and temperature is studied. The data objects are clustered on the two attributes and the probability density function is calculated based on MEP. Last, the simulation results verify the effectiveness of this method.

\section{Environment temperature and load daily law analysis}

In figure 2, the environment temperature and power data in 7th and 8th September 2011 is shown.

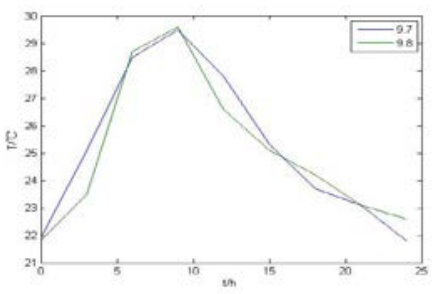

(a) The temperature data

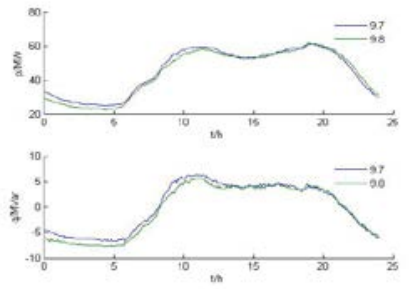

(b) The power data

Fig.2 Temperature and power data in Sep.7 and Sep.8

From the above figures, the temperature and load in different days are similar and the clustering is feasible. 


\section{Clustering and probability density function calculation}

The object attributes contain time, environment temperature, active power, reactive power, voltage and current, which can be expressed by formula (13).

$$
\text { sample }=\left[t, T, P, Q, u_{1}, i_{1}, u_{2}, i_{2}\right]
$$

Select the initial value of $K$ and calculate the distance to cluster based on $T, P$ and $Q$. And the leakage resistance is identified by the voltage and current data in the cluster with most objects.

In figure 3, the resistance data and the probability density function based on MEP is obtained. The mean value and variance is 1 and 0.01 respectively.



(a) The leakage resistance



(b) The probability density function

Fig.3 Leakage resistance and probability density function

$$
f(x)=\exp \left(-32.15+53.03 x-5.99 x^{2}-13.54 x^{3}\right)
$$

\section{Condition changes detection}

In figure 4, the cumulative probability and the leakage resistance with $1 \%$ changes caused by faults is shown. If the alarm probability value is 0.95 , the corresponding resistance value is 1.166 .

Considering rapidity and contingency, the number of new sequence and the threshold are set to be 5 and 0.2. By the method presented in this paper, the condition change is detected.

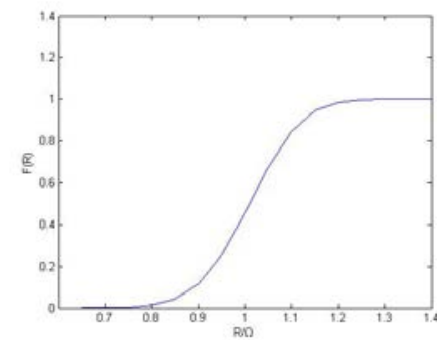

(a) The cumulative probability

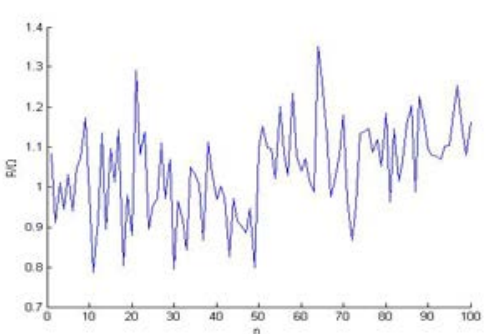

(b) The leakage resistance

Fig.4 The probability distribution function curve of leakage resistance

\section{Summary}

To detect the equipment condition changes, a method based on MEP is put forward. In this method, the influence of environment and other factors is reduced and the detection results are obtained through probability analysis, which is effective to increase accuracy.

(1) Through $K$-means clustering algorithm, the similar objects can be selected, which is effective to reduce the influence of the environment and other factors.

(2) Based on the principle of maximum entropy, the probability density function can be calculated without subjective assumptions.

(3) The condition changes are detected by the comparison of a sequence with the threshold value, which can avoid miscalculation.

\section{References}

[1] Xu Jing, Wang Jing, Gao Feng, et al. A survey of condition based maintenance technology for electric power equipments [J]. Power System Technology, 2000, 24(8) :48-52

[2] Xue Yusheng, Xu Wei, Zhaoyang Dong, et al. A review of wide area measurement system and wide area control system [J]. Automation of Electric Power Systems, 2007,31(15):1-6 
[3] Huang Jianhua, Quan Lingsan. Current status and development of condition-based maintenance of high-voltage electric power equipment in substation [J]. Automation of Electric Power System, 2001, 25(16):56-61

[4] Wang Maohai, Bao Jie, Xi Xia, et al. Online estimation of transmission line parameters based on PMU measurements [J]. Automation of Electric Power Systems, 2010, 34(1) : 25-28

[5] Bi Tianshu, Ding Lan, Zhang Daonong. Transmission line parameters identification based on moving-window TLS [J]. Journal of Electric Power Science and Technology, 2011, 26(2) : 10-15

[6] Wang Maohai, Qi Xia, Niu Siqing, et al. Online estimation of transformer parameters based on PMU measurements [J]. Automation of Electric Power Systems, 2011, 35(13) : 61-65

[7] Bi Tianshu, Liu Hao, Wu Jingtao, et al. On-line assessment on voltage consistency and frequency consistency of PMU measurement under steady state $[\mathrm{J}]$. Automation of Electric Power Systems, 2010,34(21) : 21-26

[8] Chen Jun, Yan Wei, Lu Jiangang, et al. A robust transformer parameter estimation method considering multi-period measurement random errors $[\mathrm{J}]$. Automation of Electric Power Systems, 2011, 35(2) : 28-33

[9] Cheng Tong, Yan Wei, Wen Yu, et al. Transformer parameter modifiability based transformer substation state estimation [J]. Journal of Chongqing University (Natural Science Edition), 2006, 29(3): 32-35

[10] Ni Li. Study of substation operating performance evaluation based on SCADA [D]. Jinan: Shandong University, 2011

[11]Zhang Huajun. Performance evaluation of transmission line based on SCADA [D]. Jinan: Shandong University, 2011

[12] Liu Jie. Study on key technologies of marine power equipment diagnosis system in distributed resource environment [D]. Wuhan: Wuhan University of Technology, 2010

[13] Xiao Xianyong, Ma Chao, Li Yong. Voltage sag occurrence frequency assessment caused by line faults using the maximum entropy method [J]. Proceedings of the CSEE, 2009,29(1): 87-93

[14] Xiao Xianyong, Ma Chao, Yang Honggeng, et al. Stochastic estimation of equipment sensitivity to voltage sag based on voltage sag severity index and maximum entropy principle [J]. Proceeding of the CSEE, 2009,29(31): 115-121 